\title{
Effects of health care interventions on quality of life among frail elderly: a systematized review
}

This article was published in the following Dove Medical Press journal: Clinical Interventions in Aging

\section{Jantine van}

Rijckevorsel-Scheele'

Renate CWJ Willems'

Pepijn DDM Roelofs'

Elin Koppelaar'

Robbert JJ Gobbens ${ }^{2-4}$

Marleen JBM Goumans'

'Research Centre Innovations in Care, Rotterdam University of Applied Sciences, Rotterdam, the Netherlands; ${ }^{2}$ Faculty of Health, Sports and Social Work, Inholland University of Applied Sciences, Amsterdam, the Netherlands; ${ }^{3}$ Zonnehuisgroep Amstelland, Amstelveen, the Netherlands; ${ }^{4}$ Department of Primary and Interdisciplinary Care, Faculty of Medicine and Health Sciences, University of Antwerp, Antwerp, Belgium
Correspondence: Jantine van Rijckevorsel-Scheele

Research Centre Innovations in Care, Rotterdam University of Applied

Sciences, Rochussenstraat 198, 3015 EK

Rotterdam, the Netherlands

Tel +3। 107945052

Email j.van.rijckevorsel-scheele@hr.nl
Introduction: Many health care interventions have been developed that aim to improve or maintain the quality of life for frail elderly. A clear overview of these health care interventions for frail elderly and their effects on quality of life is missing.

Purpose: To provide a systematic overview of the effect of health care interventions on quality of life of frail elderly.

Methods: A systematic search was conducted in Embase, Medline (OvidSP), Cochrane Central, Cinahl, PsycInfo and Web of Science, up to and including November 2017. Studies describing health care interventions for frail elderly were included if the effect of the intervention on quality of life was described. The effects of the interventions on quality of life were described in an overview of the included studies.

Results: In total 4,853 potentially relevant articles were screened for relevance, of which 19 intervention studies met the inclusion criteria. The studies were very heterogeneous in the design: measurement of frailty, health care intervention and outcome measurement differ. Health care interventions described were: multidisciplinary treatment, exercise programs, testosterone gel, nurse home visits and acupuncture. Seven of the nineteen intervention studies, describing different health care interventions, reported a statistically significant effect on subdomains of quality of life, two studies reported a statistically significant effect of the intervention on the overall quality of life score. Ten studies reported no statistically significant difference between the intervention and control groups.

Conclusion: Reported effects of health care interventions on frail elderly persons' quality of life are inconsistent, with most of the studies reporting no differences between the intervention and control groups. As the number of frail elderly persons in the population will continue to grow, it will be important to continue the search for effective health care interventions. Alignment of studies in design and outcome measurements is needed.

Keywords: frailty, quality of life, interventions, systematic review, multidisciplinary treatment, exercise programs

\section{Introduction}

The number of elderly people is expected to increase worldwide in the next decades. The number of persons aged 60 and above is expected to increase from 901 million in 2015 to 2.1 billion in 2050 and 3.2 billion in 2100 . The number of persons aged 80 and above is expected to increase from 125 million in 2015 to 434 million in 2050 and 944 million in $2100 .{ }^{1}$ Concurrently, the number of frail elderly will increase as well.

With the growing number of frail elderly, the concept of frailty has received more and more attention in recent years, and several definitions of frailty have been proposed. ${ }^{2-4}$ Many of these definitions are based on the theory that frailty is a multifactorial concept..$^{2-6}$ These definitions are not only based on physical components, 
resulting in difficulties with activities of daily living (ADL), but also includes components referring to psychological and social functioning of older persons. One of the definitions which describe frailty as a multifactorial concept is the definition of Gobbens et al:

Frailty is a dynamic state affecting an individual who experiences losses in one or more domains of human functioning (physical, psychological, social), which is caused by the influence of a range of variables and which increases the risk of adverse outcomes. ${ }^{3}$

The variables referred to in this definition are: sociodemographic factors (age, sex, marital status, ethnicity), socioeconomic factors (education, income), lifestyle, life events, environment and genetic factors. ${ }^{3}$

The adverse outcomes of frailty are also diverse and present on different domains. Frailty is associated with increased risk of hip fractures, disability in ADL, hospitalization, institutionalization and mortality., ${ }^{2,-9}$ Also, several studies have shown that frail elderly experience lower quality of life than elderly with less frailty. ${ }^{10-13}$ Kojima et $\mathrm{al}^{14}$ have shown in a systematic review that there is an inverse association between frailty/ pre-frailty and quality of life among community-dwelling older people. Several studies also recommend conducting intervention studies to determine if quality of life in this frail population could be improved or maintained. ${ }^{15,16}$

Therefore, many health care interventions have been developed aiming to improve or maintain quality of life of frail elderly. These health care interventions are of a diverse nature, including all interventions with the aim to maintain or improve the health of the body and/or mind. Examples of these health care interventions are multidisciplinary interventions or physical exercise interventions. Nonetheless, a clear overview of these health care interventions for frail elderly and their effects on quality of life is missing. The objective of this review is therefore to provide a systematic overview of the effect of health care interventions on quality of life of frail elderly.

\section{Material and methods Study design}

Systematized review according to the description of Grant et al. ${ }^{17}$ This included a systematic research process. The presentation of the results is narrative with tabular accompaniment. ${ }^{17}$ The review protocol was not registered.

\section{Eligibility criteria}

Type of studies: All clinical trials with a quantitative design.
Type of participants: Frail elderly identified with any frailty measurement instrument that assessed frailty on different domains.

Type of interventions: All types of health care interventions. These interventions were compared with a control intervention, either usual care or another intervention.

Type of outcome measurement: Quality of life.

Inclusion criteria were: 1) population of frail elderly; 2) measurement of frailty described in the study; 3) study about health care interventions; 4) study described the effect of the intervention on quality of life; 5) study designed as a randomized controlled trial, quasi-randomized controlled trial, controlled clinical trial or clinical trial; and 6) study was written in English, Dutch, German, or French.

\section{Search strategy}

Studies were identified by searching the Embase, Medline (OvidSP), Cochrane Central, Cinahl, PsycInfo and Web of Science databases from their inception up to and including November 2017. Keywords used were "frail elderly" (or frail* in a combination with eg, old or aged) and "quality of life" (or related terms such as QoL or HRQL). The complete search strategy for Embase and Medline (OvidSP) is presented in Supplementary material. This search strategy was adapted for the other databases. The adapted search strategies are available on request. The reference lists of all relevant reviews and potentially relevant studies were screened for additional studies. When relevant study designs or congress abstracts were found, the databases were searched for articles describing results of these studies more completely.

\section{Study selection}

Titles and abstracts of potentially relevant studies were screened by JvR or RW based on the inclusion criteria. Both authors independently read all full text articles reporting potentially relevant studies to make the final selection, using the same inclusion criteria. Disagreement was solved by discussion and consensus by the two reviewers. In case of persistent disagreement, a third reviewer could be consulted.

\section{Risk of bias in individual studies}

Risk of bias of the included studies was assessed using the "Cochrane collaboration's tool for assessing risk of bias". ${ }^{18}$ This list contains seven items: random sequence generation, allocation concealment, blinding of participants and personnel, blinding of outcome assessment, incomplete outcome data, selective reporting, and other sources of bias. Each item was rated as low risk of bias, high risk of bias, or unclear. The methodological quality of the selected studies 
was independently assessed by two authors (JvR, RW). Disagreement was resolved by discussion and consensus of the two reviewers. In case of persistent disagreement, a third reviewer could be consulted.

\section{Data extraction and analysis}

The following data were extracted from the included studies: study population (age, setting, number of participants), measurement of frailty, study characteristics (design, length of follow-up), type of intervention, control treatment and outcome: quality of life. If provided, the mean scores and SD for quality of life in the intervention and control groups were extracted. Given outcomes were extracted when mean scores and SD were not presented. One author (JvR or RW) performed the data extraction, which was checked by another author (JvR or RW). The authors of an included study were contacted if more information was required.

The outcomes of the included studies were described.

\section{Results Literature search}

The literature search resulted in 4,853 potentially relevant articles (Figure 1). After screening titles and abstracts, 250 potentially eligible articles were identified. Most of the articles excluded at this stage did not report about an intervention study $(n=2,107)$ or the study population was not frail $(n=513)$. This means that frailty either was not measured at all or was measured with an instrument that only assessed ADL. After reviewing the literature lists of potentially relevant studies, two studies were added for full text screening. Thus, in total 252 full text articles were evaluated. Twenty-three were included in this review. Most of the excluded articles reported a study that did not describe the results of a health care intervention $(n=92)$, did not measure frailty $(n=48)$, or did not measure quality of life $(n=28)$. The 23 included articles described the results of 19 different interventions studies. Several articles described different follow-up periods from

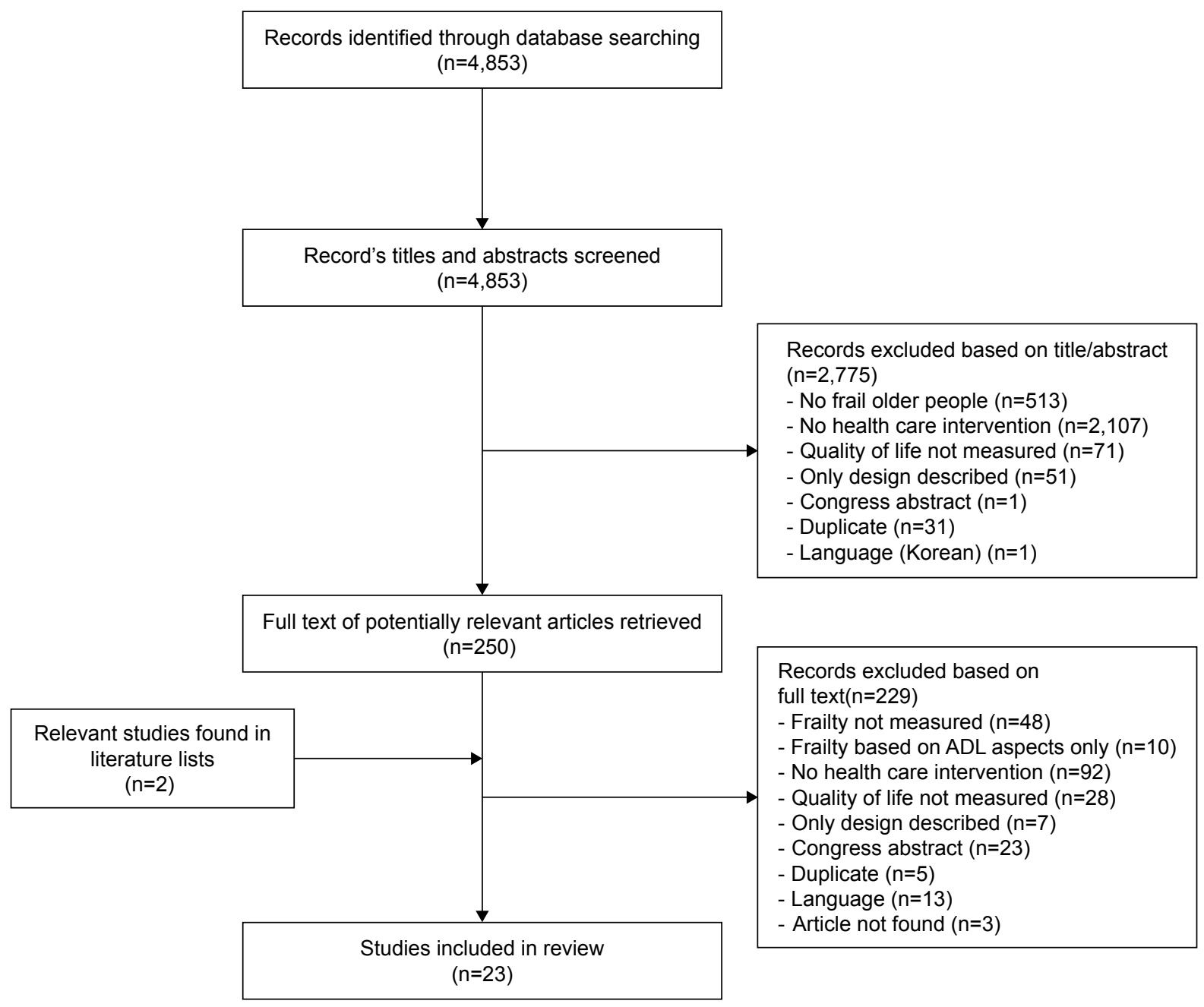

Figure I Study selection flowchart.

Abbreviation: ADL, activities of daily life. 
the same intervention study, ${ }^{19-24}$ and two articles described different aspects of the same intervention study. ${ }^{25,26}$

\section{Methodological quality}

An overview of the methodological quality of the intervention studies is presented in Figure 2.

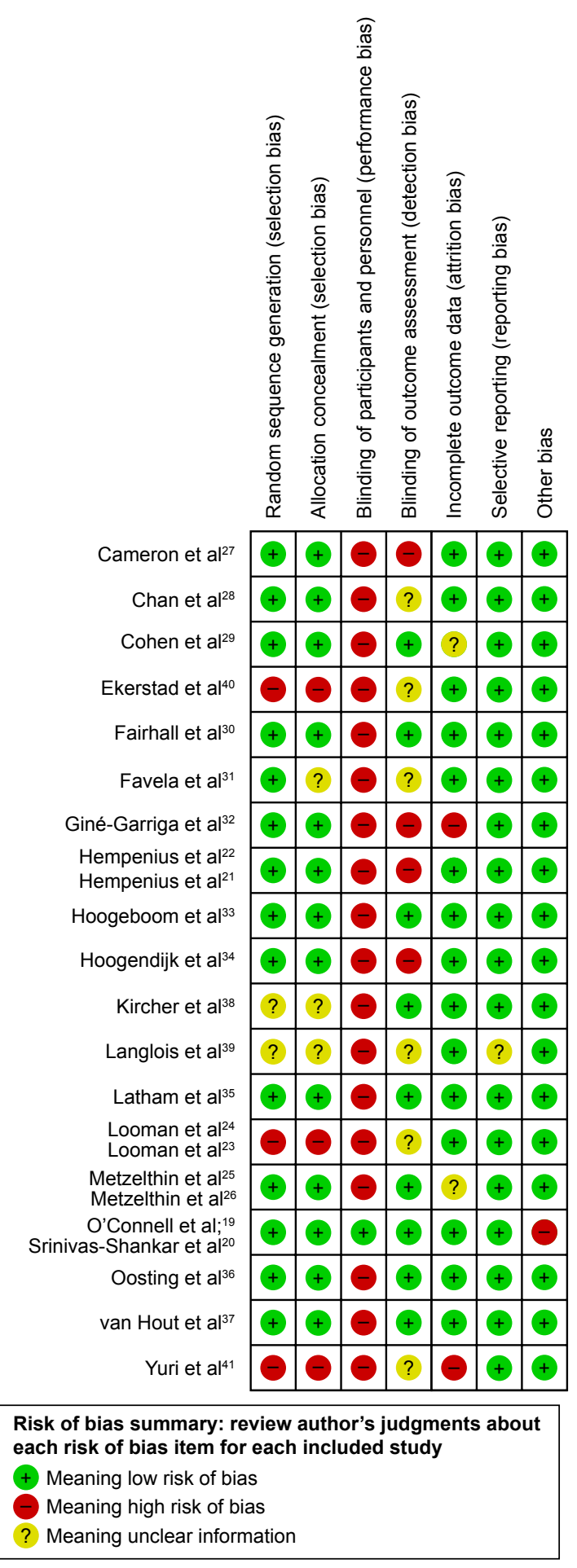

Figure 2 Methodological quality of the included studies, using the Cochrane collaboration's tool for assessing risk of bias.
Fourteen of the nineteen included intervention studies used an adequate method for random sequence generation. ${ }^{19-22,25-37}$ Two of the nineteen studies provided insufficient information about the sequence generation process to permit judgment of low or high risk. ${ }^{38,39}$ The only study in which participants, personnel as well as the outcome assessor, were blinded from knowledge of which intervention a participant received was the one described in the articles of O'Connell et al ${ }^{19}$ and Srinivas-Shankar et al. ${ }^{20}$ In this study, the use of a placebo testosterone gel served as control intervention. Eight other studies did blind the outcome assessor. ${ }^{25,26,29,30,33,35-38}$ In the study of Cameron et al, ${ }^{27}$ outcome assessors were blinded, but many participants disclosed their treatment status to the outcome assessor. The risk of incomplete outcome data was unclear in two studies due to lack of information. ${ }^{25,26,29}$ The risk of incomplete outcome data was high in the study of Giné-Garriga et $\mathrm{al}^{32}$ and the study of Yuri et $\mathrm{al}^{41}$ due to the high percentage and imbalance of loss to follow up, both in the control group and in the intervention group, and the lack of a sensitivity analysis. Other bias was high in the study of O'Connell et $\mathrm{al}^{19}$ and Srinivas-Shankar et $\mathrm{al}^{20}$ because they were sponsored by the pharmaceutical industry. Figure 3 shows the risk of bias summery, presenting the review authors' judgments about each risk of bias item presented as percentages across all included studies. This indicates that across the studies there is a high risk of performance bias because many studies did not blind the participants and/or the personnel.

\section{Characteristics of the included studies}

An overview of the characteristics and outcomes of the included studies is presented in Table 1. Populations and settings, frailty measurement instruments, quality of life measurements instruments and the types of intervention analyzed varied.

\section{Population and settings}

All studies had a minimum age limit, ranging from 60 to 75 years. Study participants were recruited from general practices, ${ }^{19,20,23-26,32,34,37}$ hospitals, ${ }^{21,22,33,36,38,40}$ rehabilitation centers, ${ }^{27,30,35}$ veterans affairs medical centers, ${ }^{29}$ neighborhood elderly centers, ${ }^{28}$ health insurance database supplied by the Mexican Social Security Institute, ${ }^{31}$ a certain city, ${ }^{41}$ or unknown. ${ }^{39}$

\section{Frailty measurements}

Different measurement instruments were used by the included studies to identify frail people. Validated instruments included the criteria of Fried which was used to identify frail people in two of the included studies, ${ }^{19,20}$ the Groningen Frailty Indicator (GFI) was used in six of the 


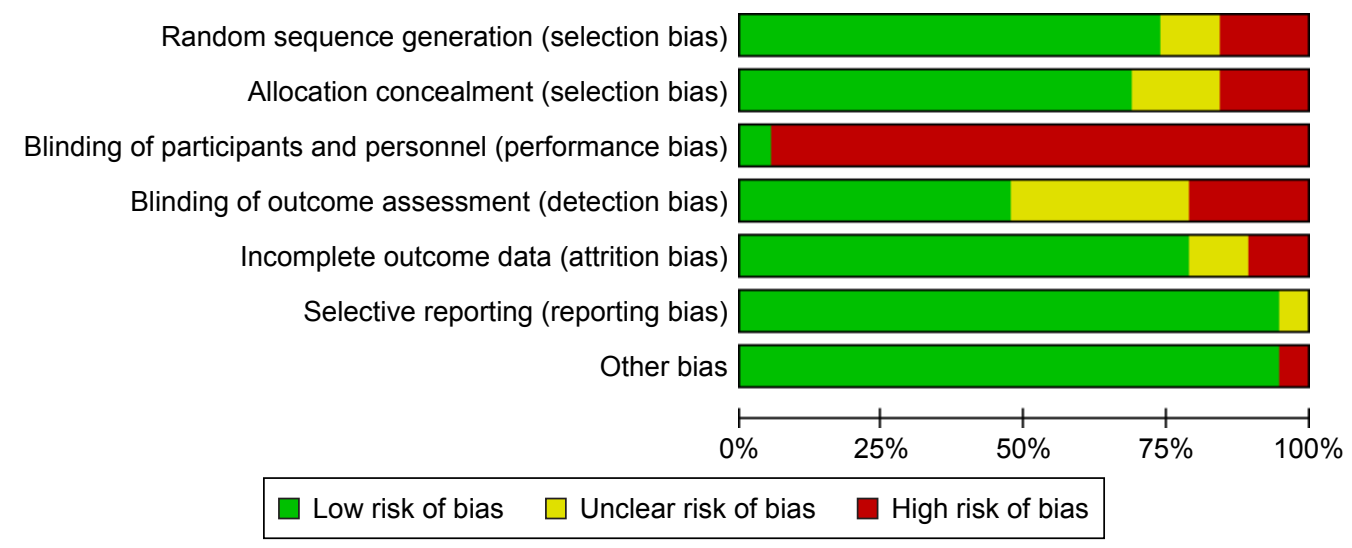

Figure 3 Risk of bias graph: review authors' judgments about each risk of bias item presented as percentages across all included studies.

included studies. ${ }^{21-26}$ Other validated measurement instruments which were used by the included studies were the Tilburg Frailty Indicator (TFI), ${ }^{28}$ the Canadian Study of Health and Aging Clinical Frailty Scale, ${ }^{33}$ the criteria proposed by Lachs et al, ${ }^{38}$ the Frail Elderly Support research group screening instrument, ${ }^{40}$ the PRISMA-7, ${ }^{34}$ the Kihon checklist, ${ }^{41}$ and the frailty index "Identification of Seniors At Risk (ISAR)". ${ }^{36}$ The authors of other studies used newly developed frailty measurements ${ }^{29,31,35,37}$ or combined existing frailty measurements with their own selection criteria. ${ }^{32,39}$

\section{Quality of life instruments}

The different instruments used to determine quality of life were: EuroQol (EQ)-5D, ${ }^{23,25-27,30,34}$ EQ-6D, ${ }^{24}$ Short Form (SF)-36. ${ }^{21,22,29,31,35,37}$ SF-12, ${ }^{32,34}$ RAND-36, ${ }^{24}$ Aging Males' Symptom scale, ${ }^{19,20}$ Hip Osteoarthritis Outcome Score, ${ }^{33,36}$ Quality of Life Philadelphia Geriatric Center Morale Scale (PGCMS), ${ }^{38}$ the Quality of Life Systemic Inventory questionnaire, ${ }^{39}$ the WHO Quality of LifeBREF (WHOQOL-BREF), ${ }^{28}$ the Health Utilities Index-3 (HUI-3), ${ }^{40}$ the Investigating Choice Experiments for CAPability measure for Older people (ICECAP) ${ }^{24}$ and a 5-point Likert scale. ${ }^{41}$

\section{Type of interventions}

Nine studies examined the effect of interventions based on a multidisciplinary treatment program with geriatric evaluation vs usual care. ${ }^{21-27,29,30,34,38,40}$ Five studies examined the effect of exercise programs vs usual care,,$^{32,33,35,36,39}$ one study a combination of vitamin D and exercise vs a placebo tablet and social home visits ${ }^{35}$ and one study a preventive care program which included physical exercise classes, oral care and nutrition education. ${ }^{41}$ Other interventions described were testosterone gel vs placebo, ${ }^{19,20}$ acupuncture intervention vs waiting list ${ }^{28}$ and nurse home visits with or without alert buttons vs usual care. ${ }^{31,37}$

\section{Effect of health care intervention on quality of life among frail elderly}

Findings on the effect of the health care intervention on quality of life are also presented in Table 1. Based on the similarity of the intervention and the number of studies describing the intervention, the results of the included studies will be described in three subgroups: multidisciplinary treatment, exercise programs and other intervention.

\section{Multidisciplinary treatment program with geriatric evaluation}

Five of the nine studies which examined the effect of a multidisciplinary treatment program with geriatric evaluation reported no statistically significant differences concerning quality of life between the intervention and control groups. ${ }^{25-27,30,34,38}$ Hempenius et al ${ }^{21}$ reported no significant differences between groups at three months after discharge and no significant differences between groups for most SF-36 subscales at discharge, except for the SF-36 subscale bodily pain (OR $0.49,95 \%$ CI: $0.29-0.82$ ). ${ }^{22}$ Looman et $\mathrm{al}^{23}$ reported a statistically significant difference between groups in the attachment dimension of the ICECAP after 3 and 12 months follow-up: frail elderly in the control group perceived receiving lesser amounts of love and friendship than desired, whereas the intervention group was stable on this dimension. ${ }^{23,24}$ Cohen et al ${ }^{29}$ reported statistically significant differences between groups in different dimensions of the SF-36 by comparing the mean change in scores at different time points, for example at baseline and discharge, of the intervention and control groups. In their study, inpatient geriatric care had a positive effect on the following domains at discharge: physical function (mean change in score of the intervention group: -1.5 , mean change in score of the control group: $-5.4, P=0.006$ ), bodily pain ( $P=0.001)$, energy $(P=0.01)$, and general health $(P=0.006)$. The effect on the 


\begin{tabular}{|c|c|c|c|}
\hline & 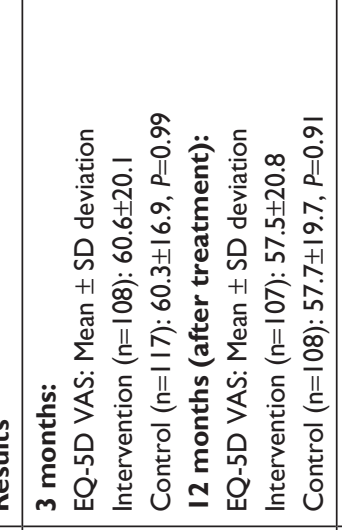 & 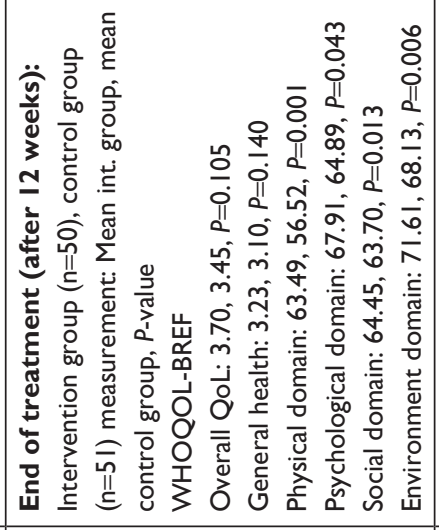 & 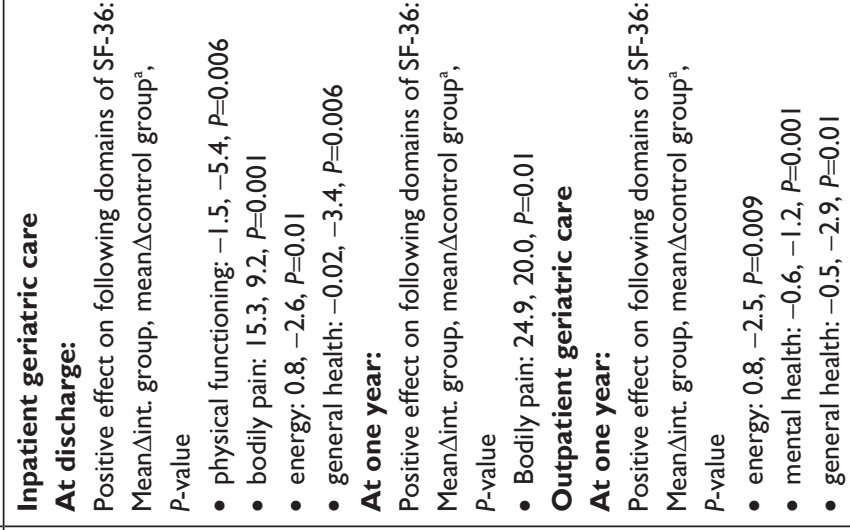 \\
\hline & 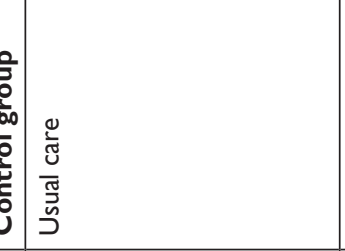 & 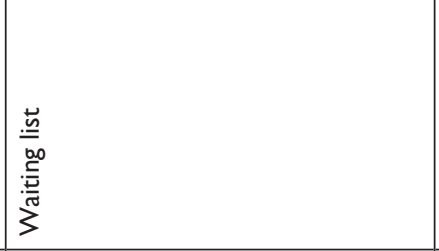 & 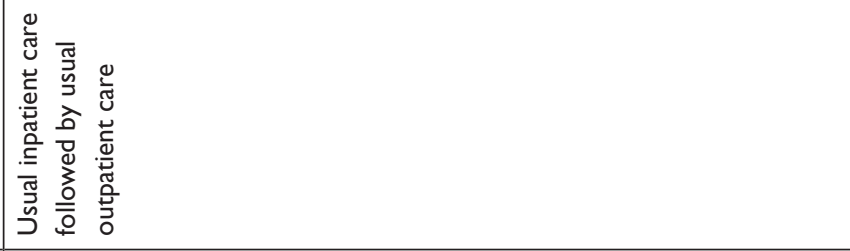 \\
\hline & 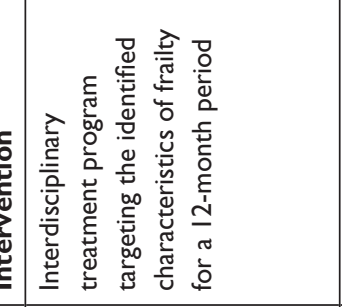 & 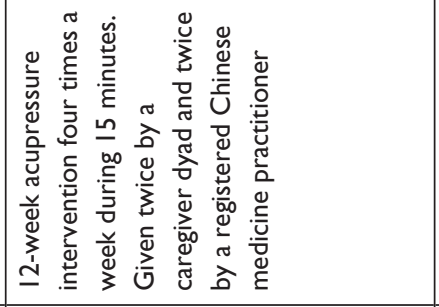 & 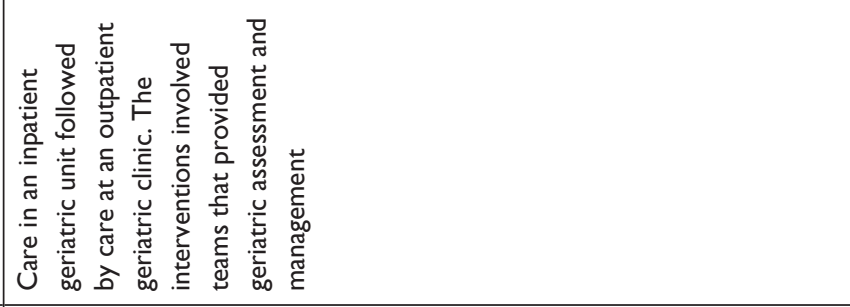 \\
\hline & 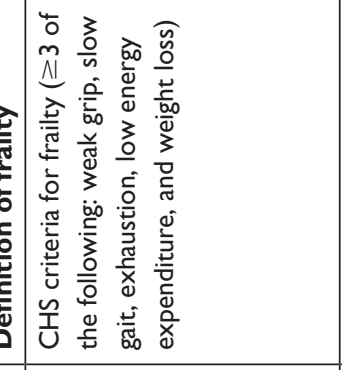 & 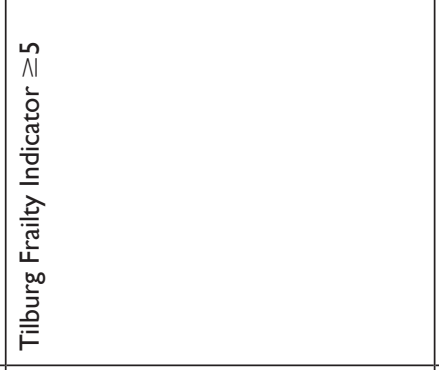 & 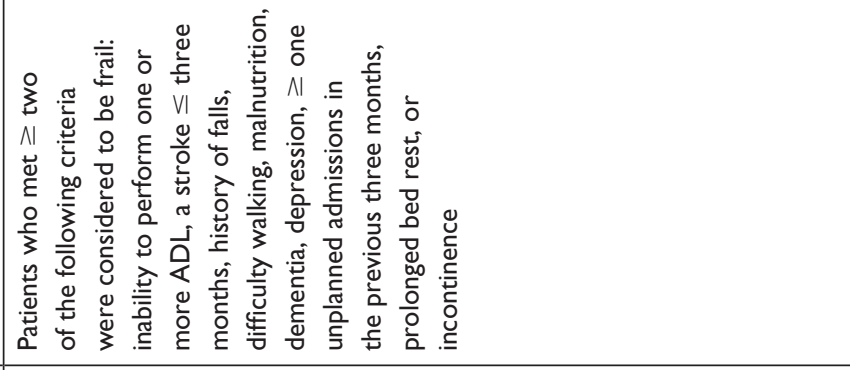 \\
\hline & 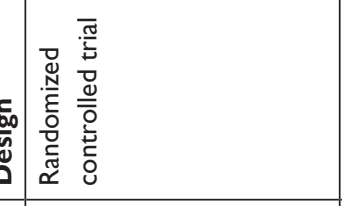 & 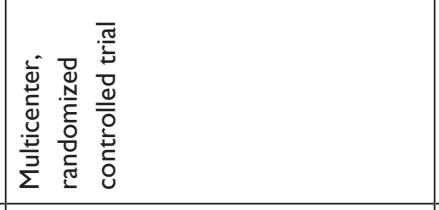 & 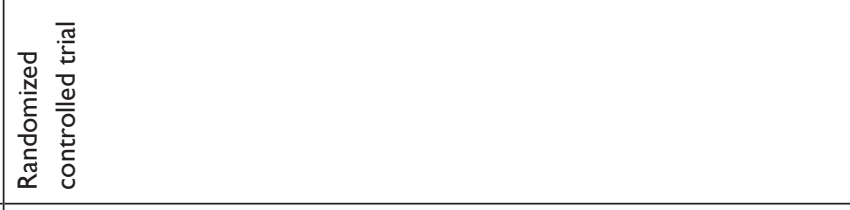 \\
\hline & 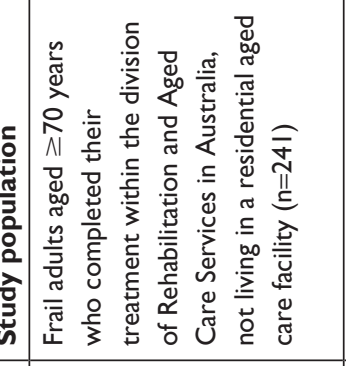 & 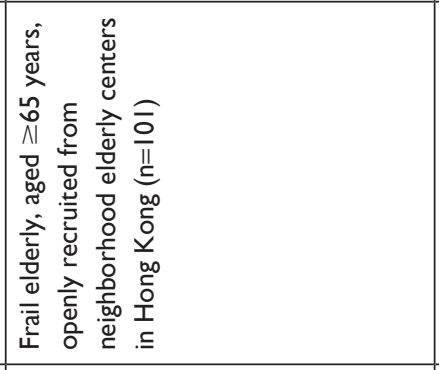 & 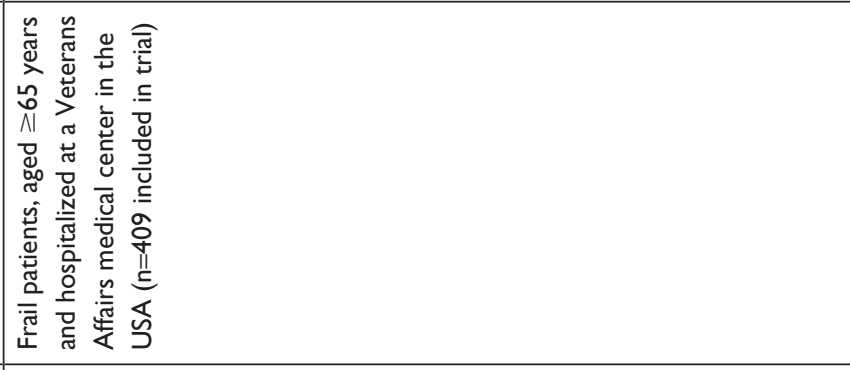 \\
\hline & 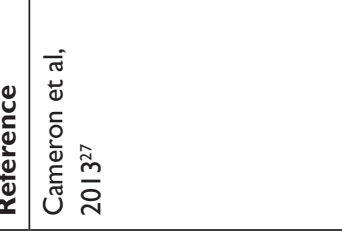 & 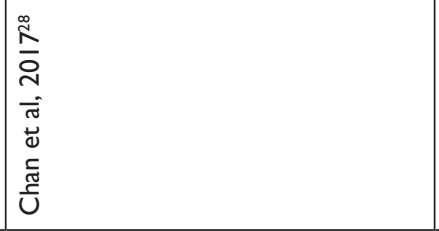 & 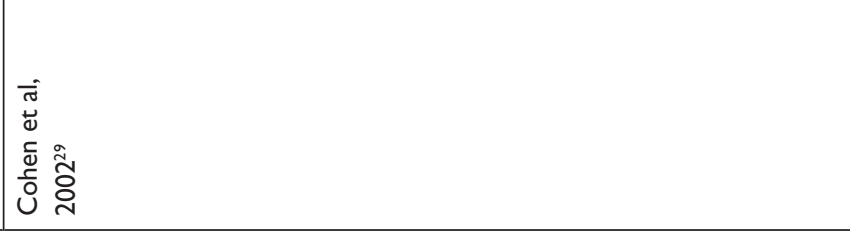 \\
\hline
\end{tabular}




\begin{tabular}{|c|c|c|c|}
\hline \multirow[t]{7}{*}{ 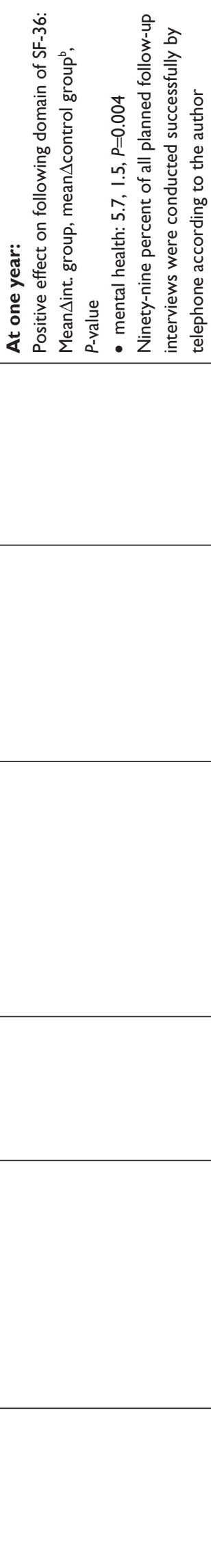 } & 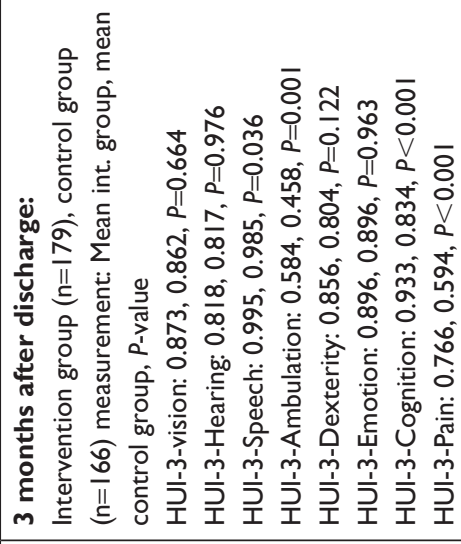 & 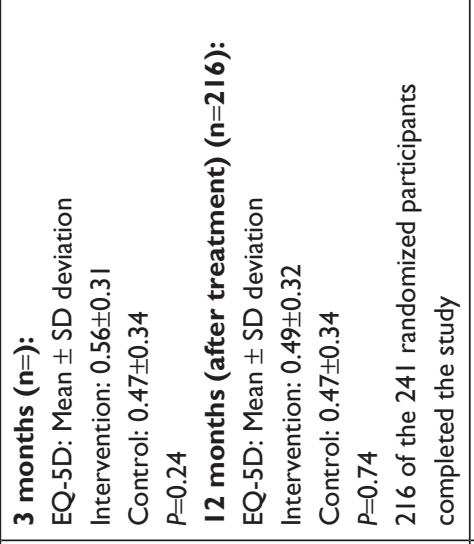 & 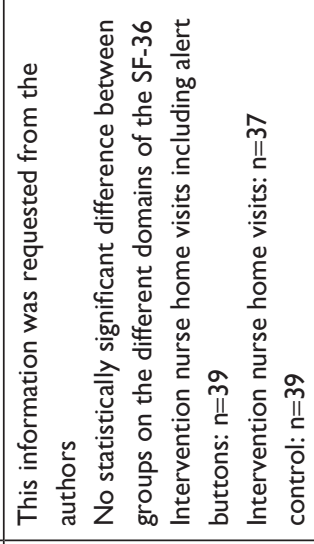 \\
\hline & 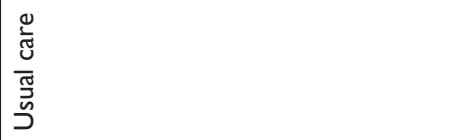 & 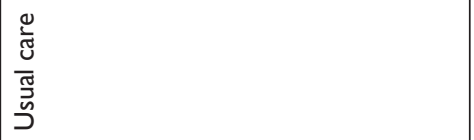 & 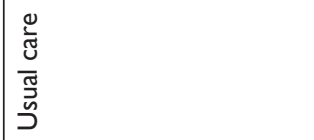 \\
\hline & 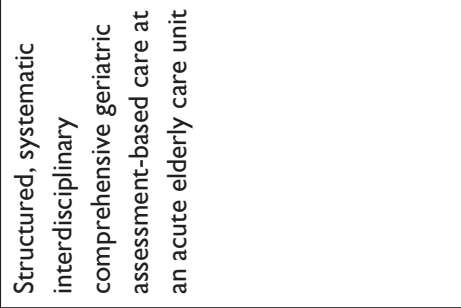 & 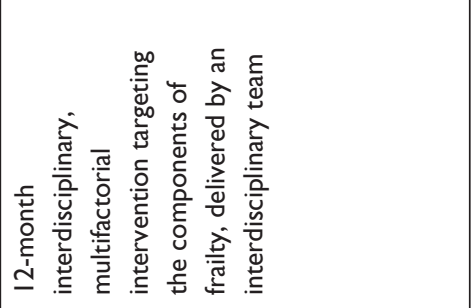 & 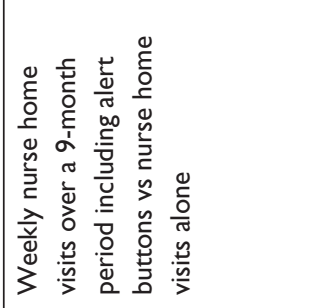 \\
\hline & 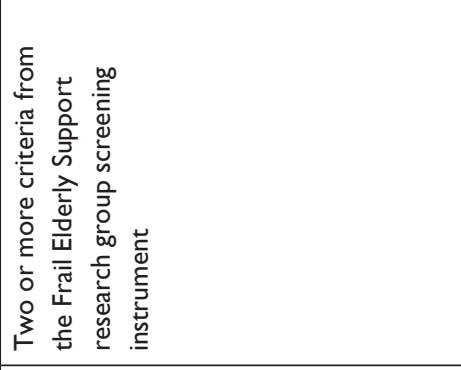 & 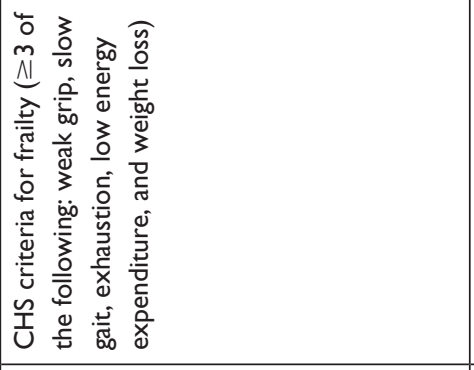 & 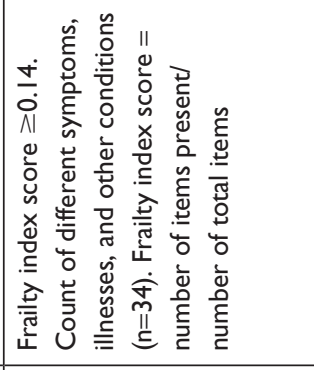 \\
\hline & 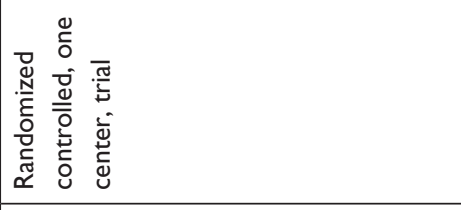 & 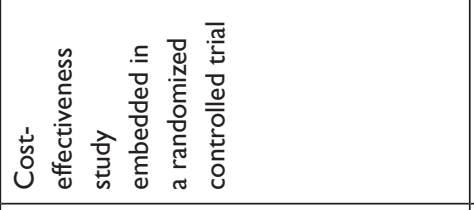 & 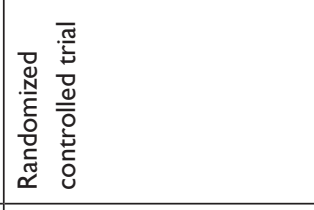 \\
\hline & 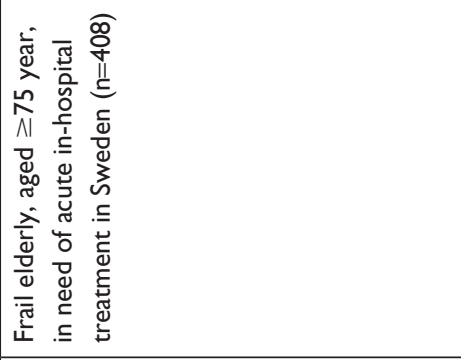 & 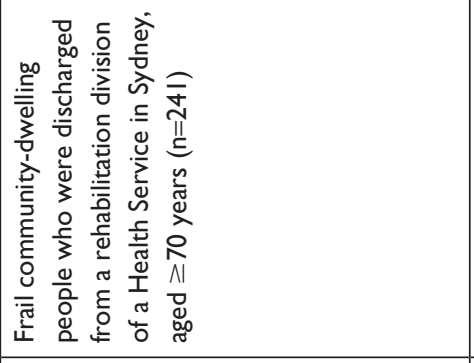 & 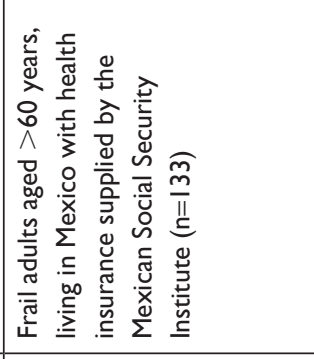 \\
\hline & 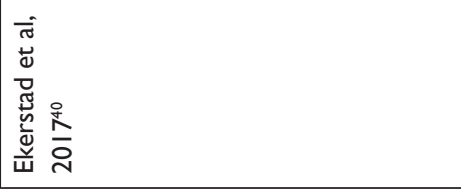 & 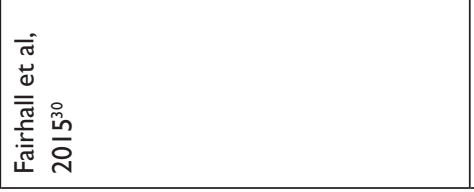 & 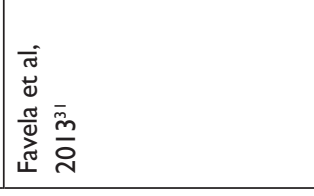 \\
\hline
\end{tabular}




\begin{tabular}{|c|c|c|c|c|}
\hline & 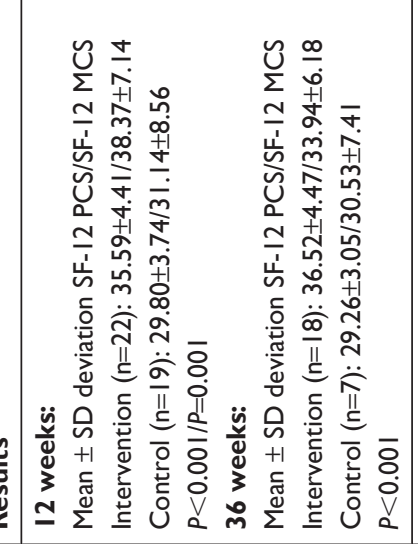 & 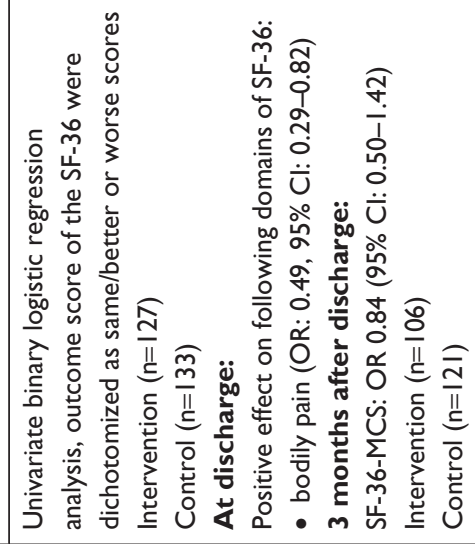 & 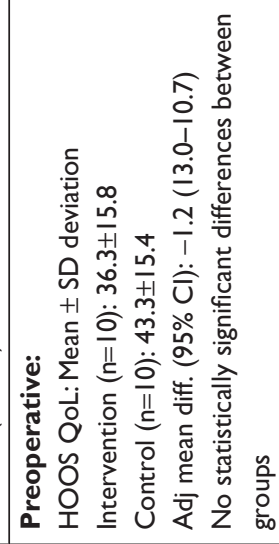 & 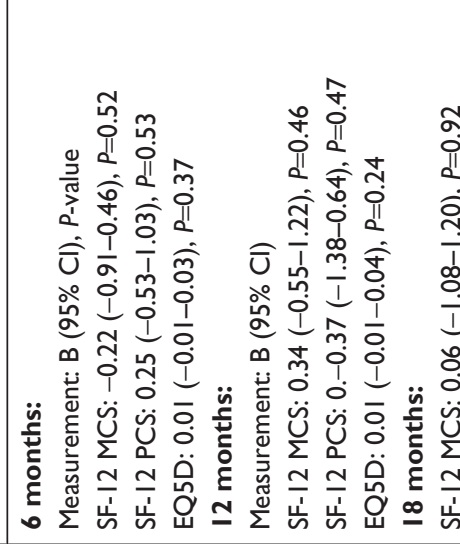 \\
\hline & 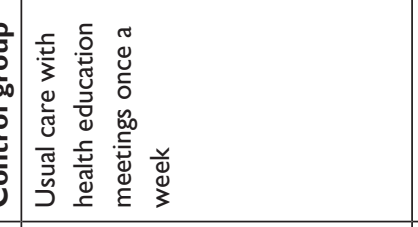 & 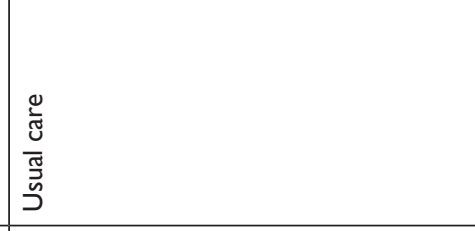 & 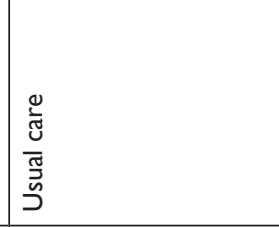 & 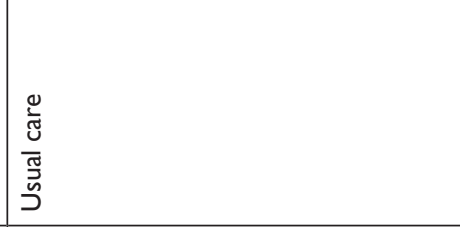 \\
\hline & 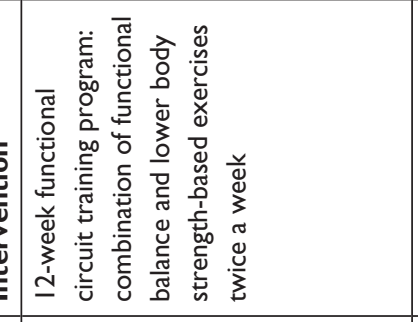 & 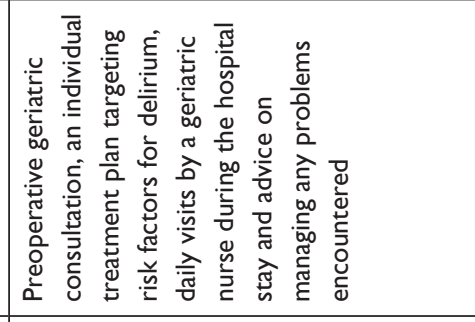 & 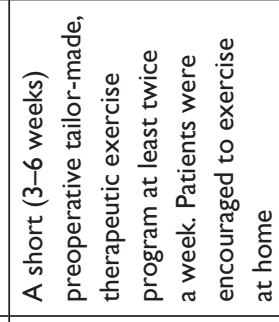 & 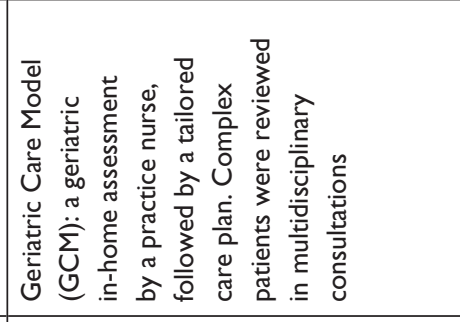 \\
\hline & 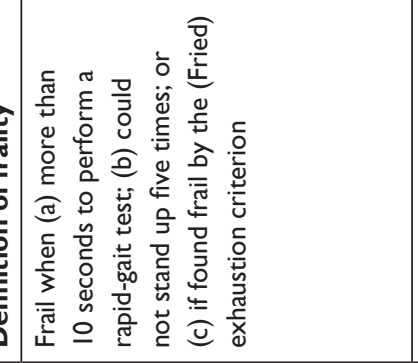 & 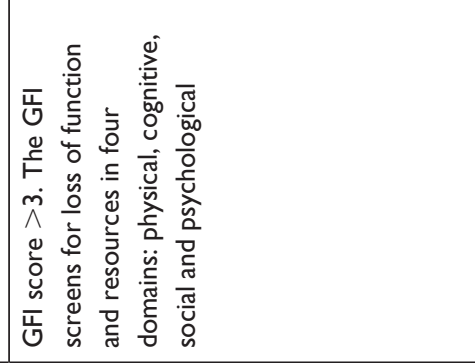 & 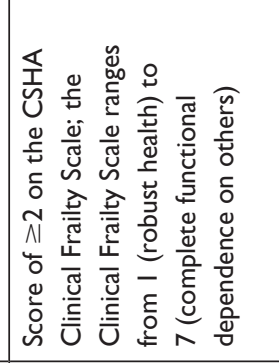 & 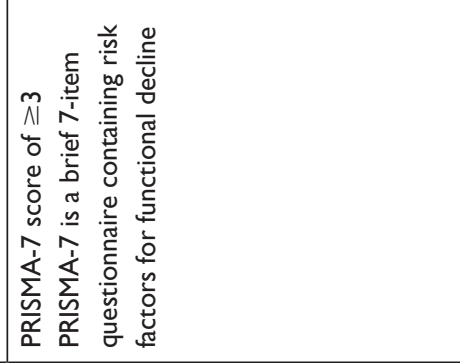 \\
\hline & 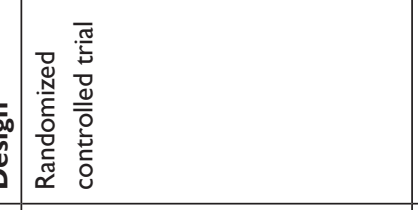 & 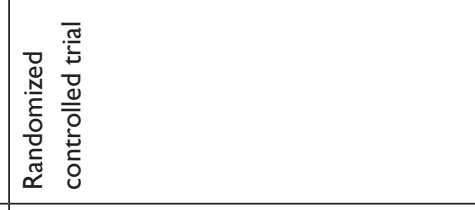 & 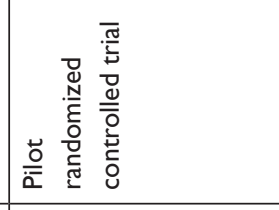 & 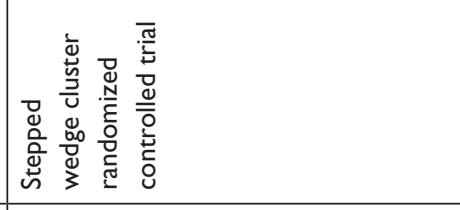 \\
\hline & 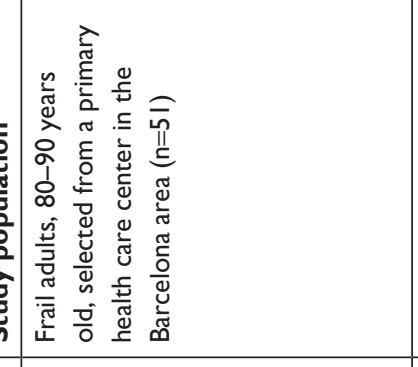 & 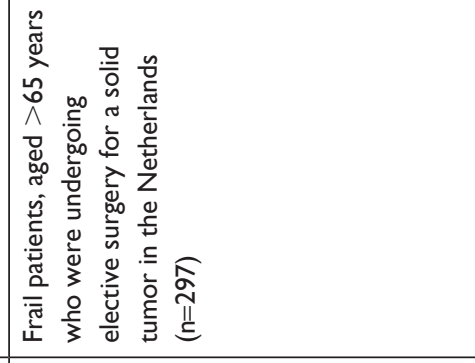 & 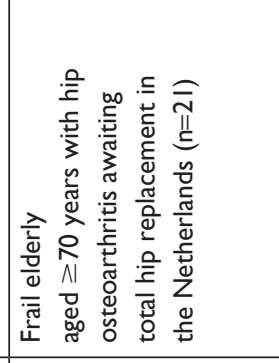 & 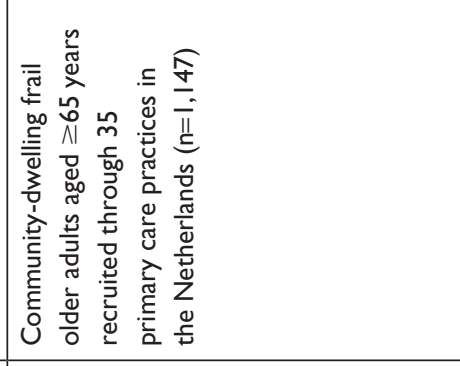 \\
\hline & 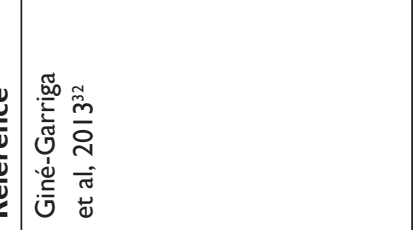 & 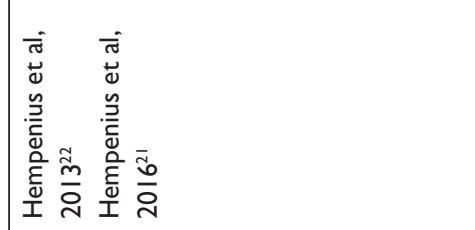 & 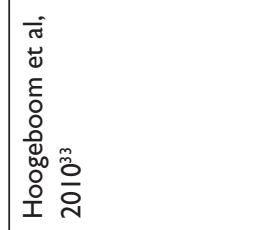 & 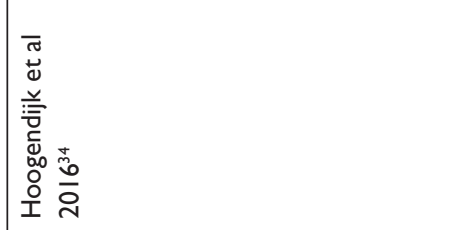 \\
\hline
\end{tabular}




\begin{tabular}{|c|c|c|}
\hline 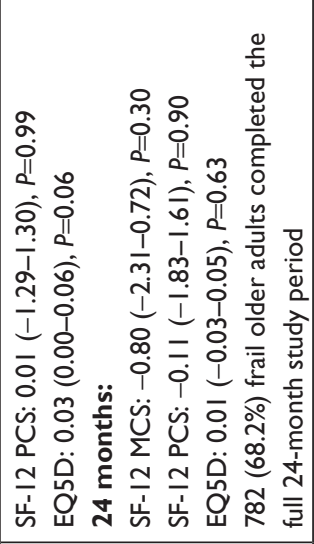 & 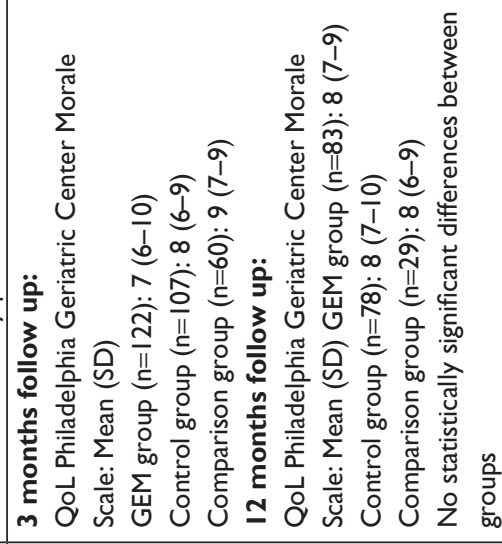 & 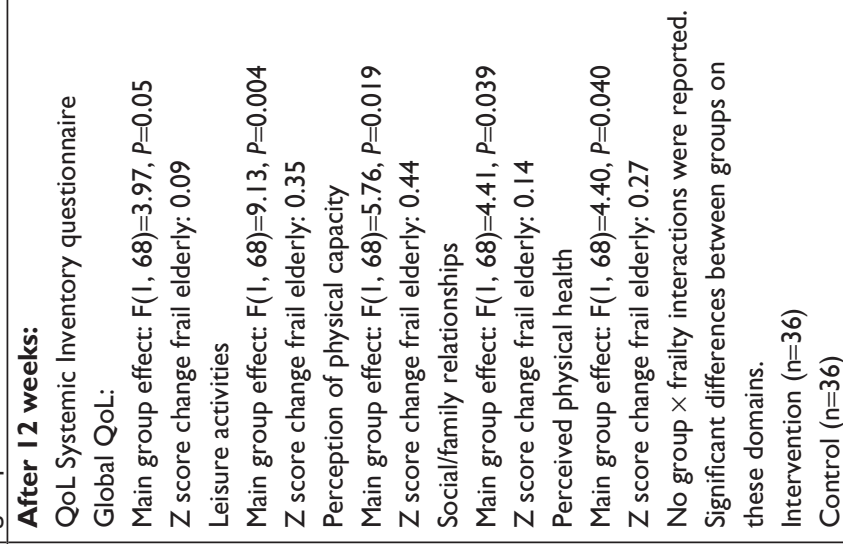 \\
\hline & 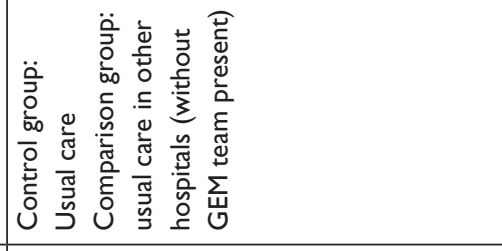 & 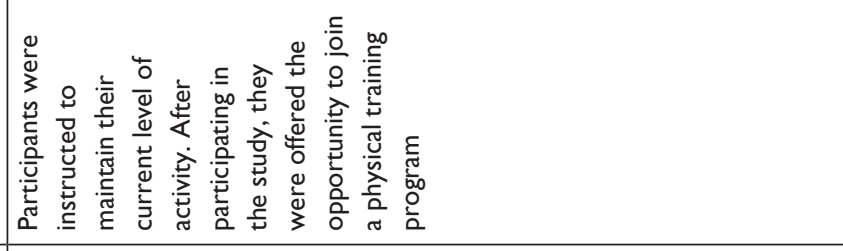 \\
\hline & 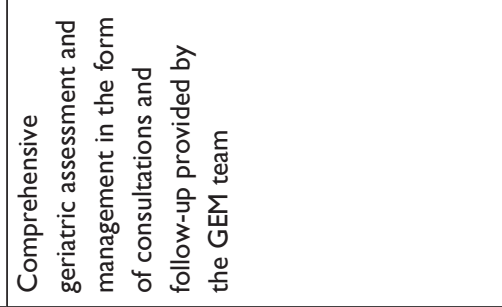 & 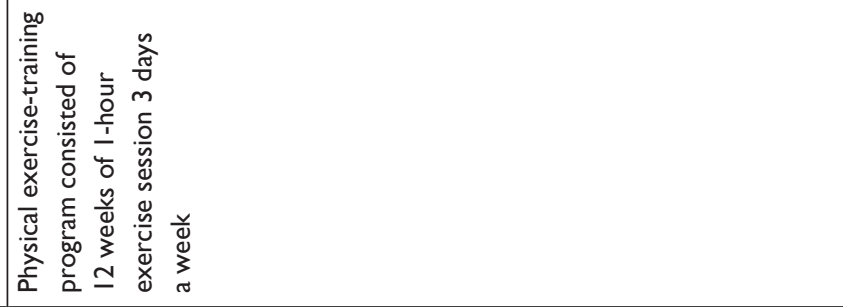 \\
\hline & 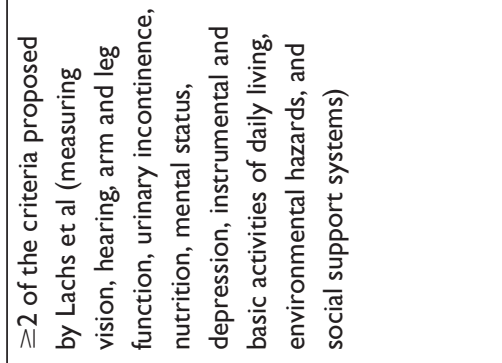 & 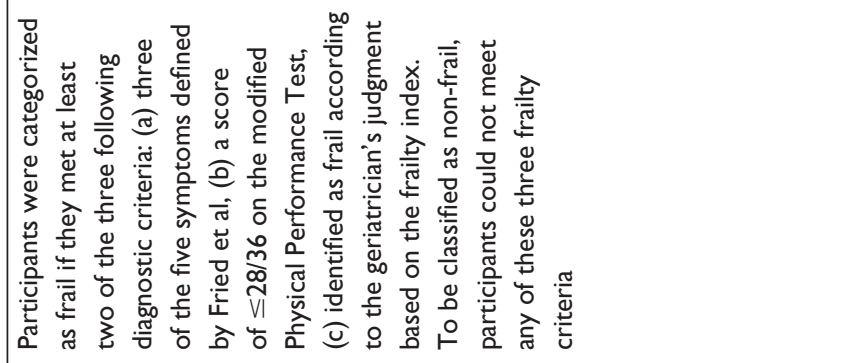 \\
\hline & 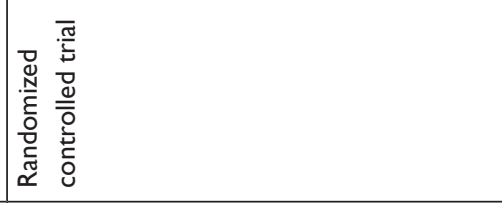 & 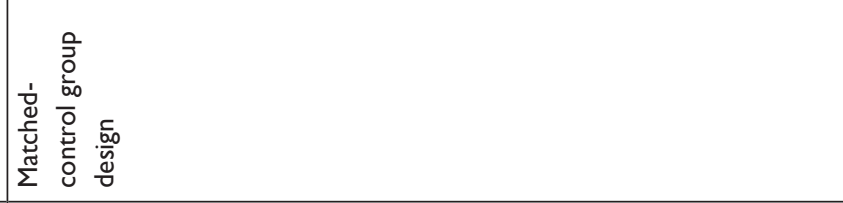 \\
\hline & 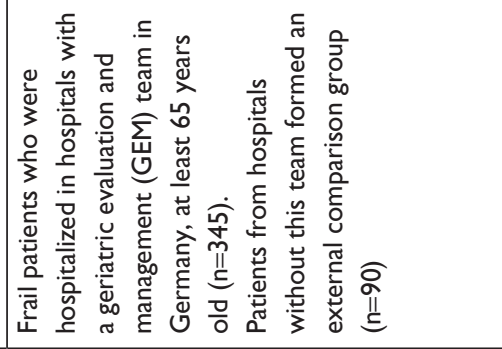 & 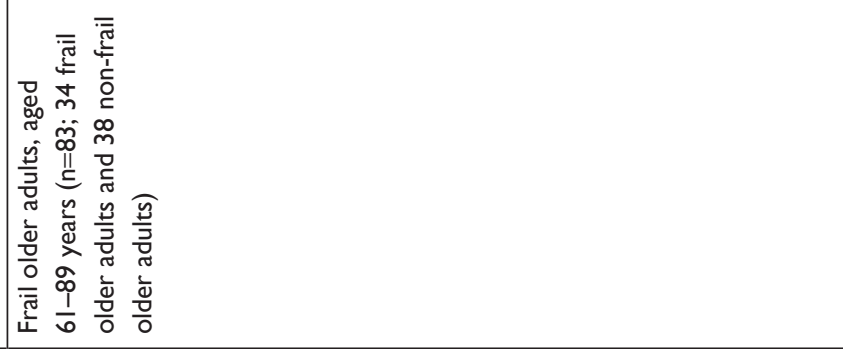 \\
\hline & 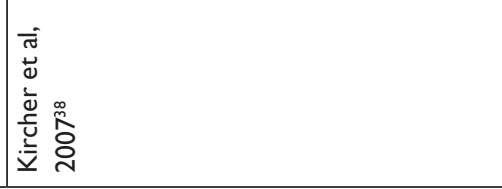 & 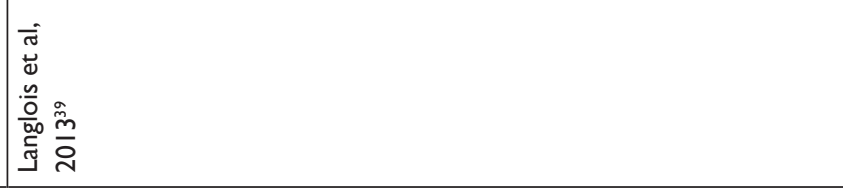 \\
\hline
\end{tabular}




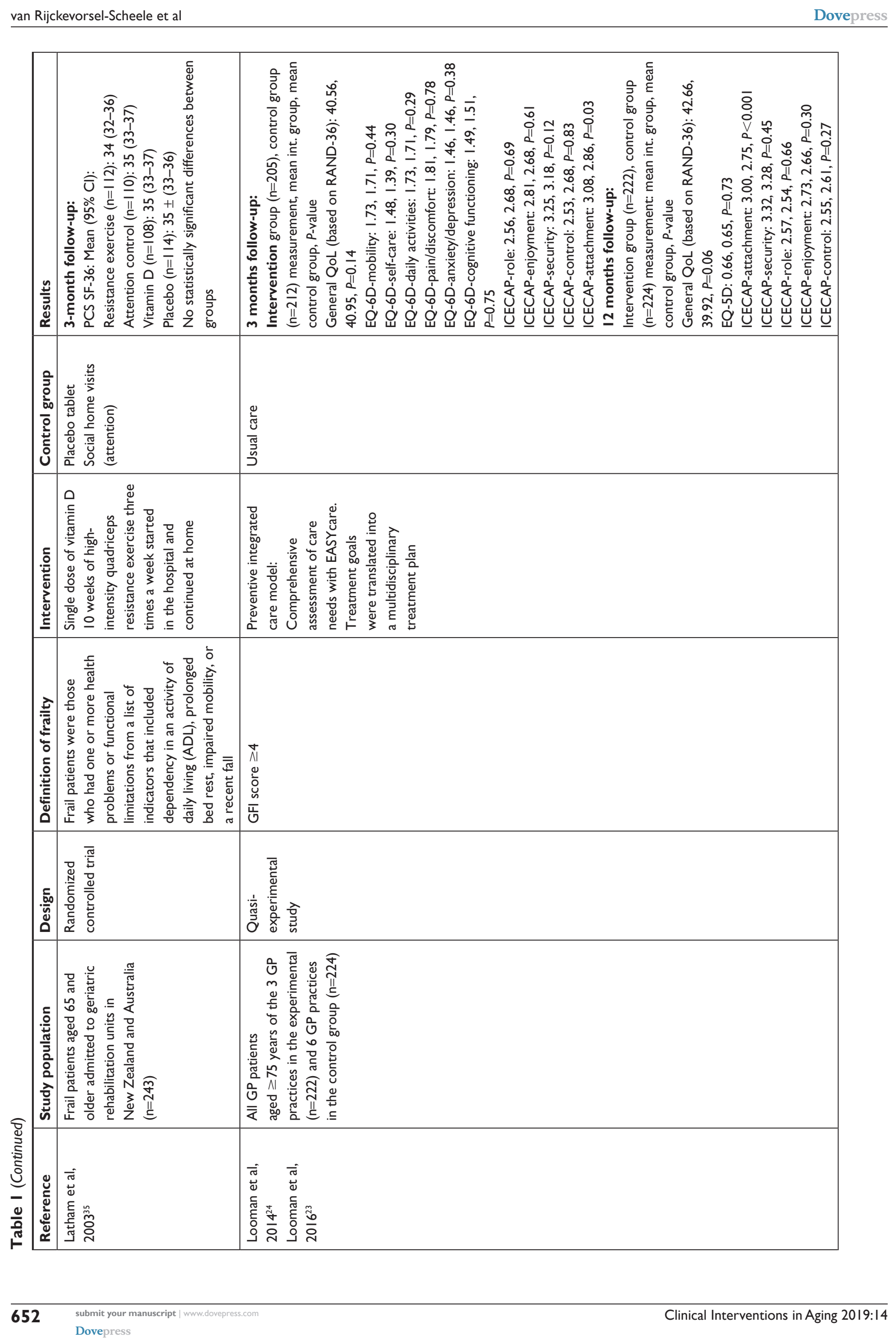




\begin{tabular}{|c|c|c|}
\hline 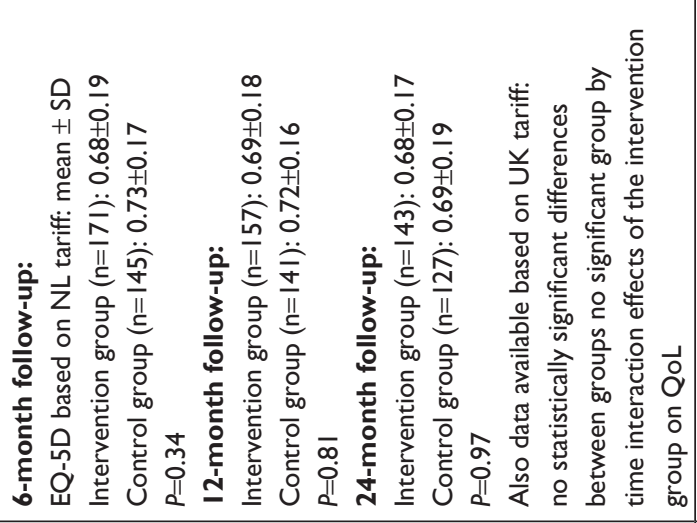 & 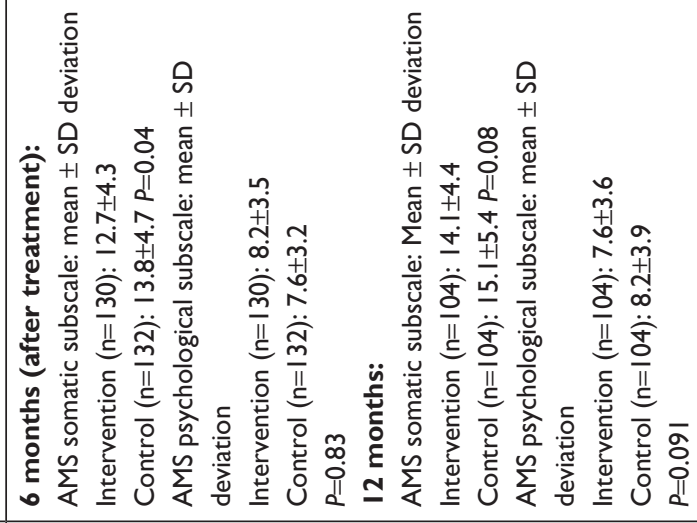 & 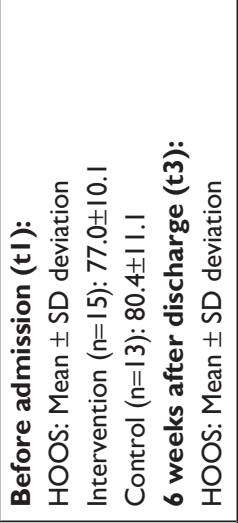 \\
\hline & & 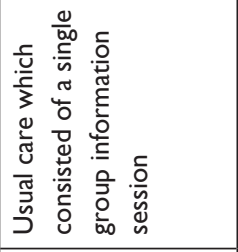 \\
\hline 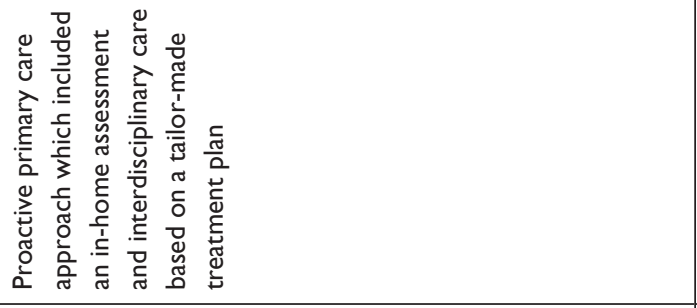 & 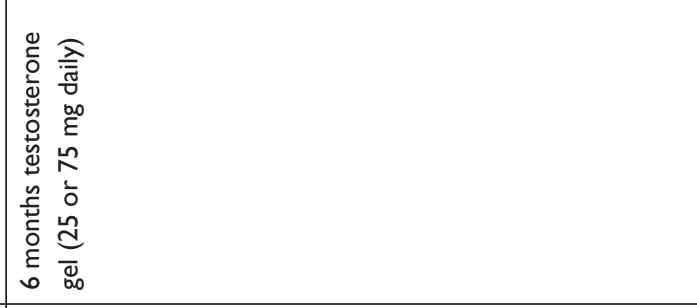 & 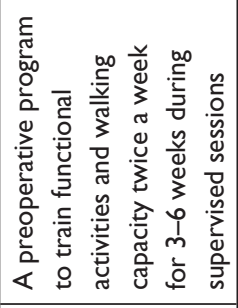 \\
\hline 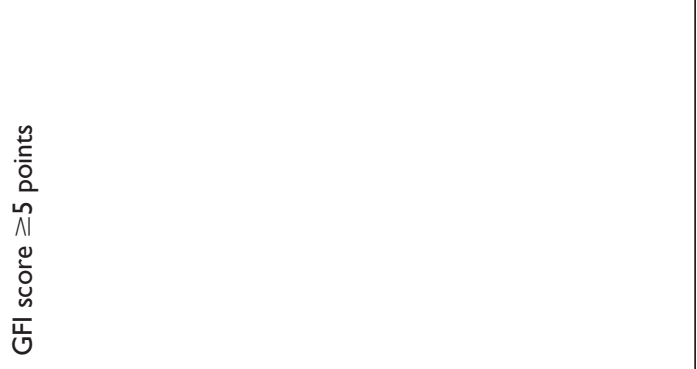 & 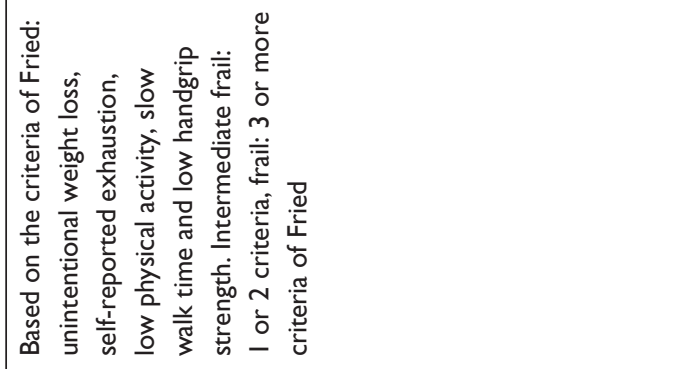 & 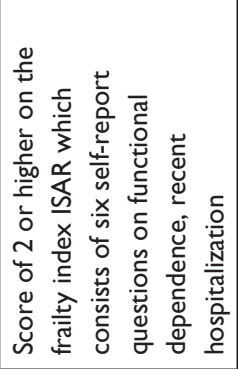 \\
\hline 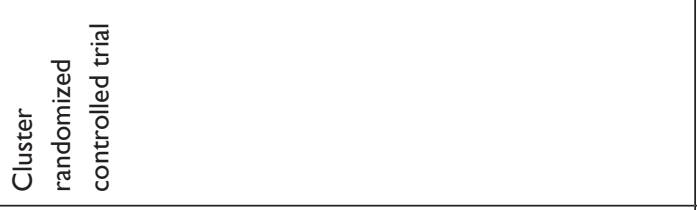 & 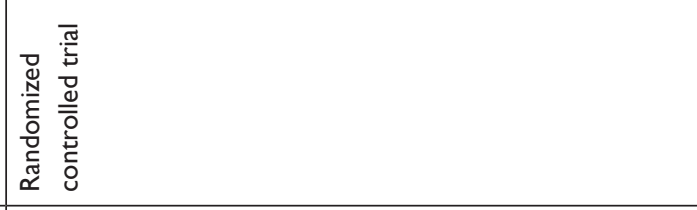 & 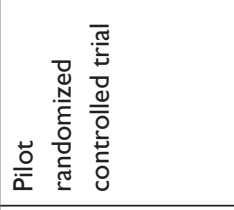 \\
\hline 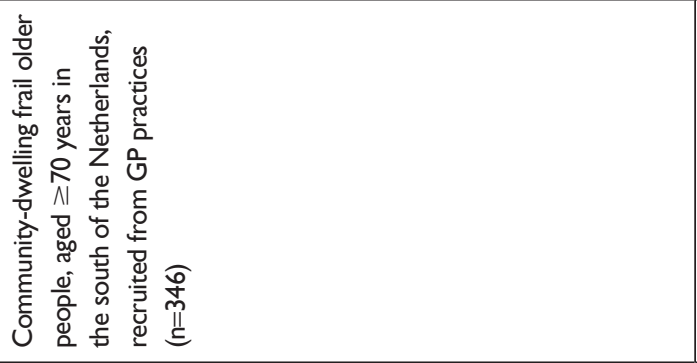 & 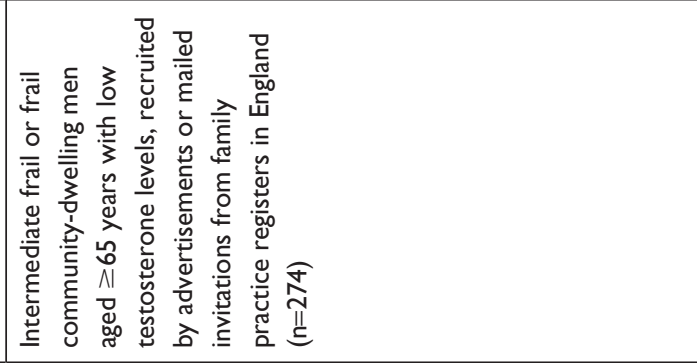 & 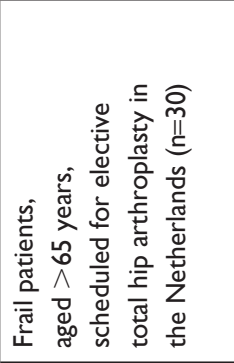 \\
\hline 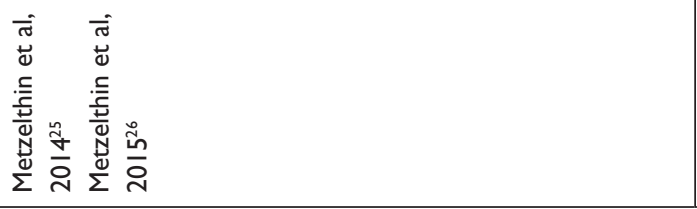 & 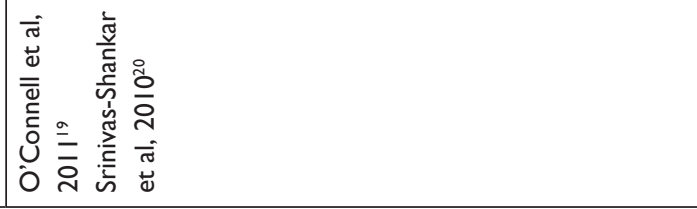 & 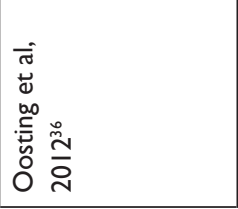 \\
\hline
\end{tabular}




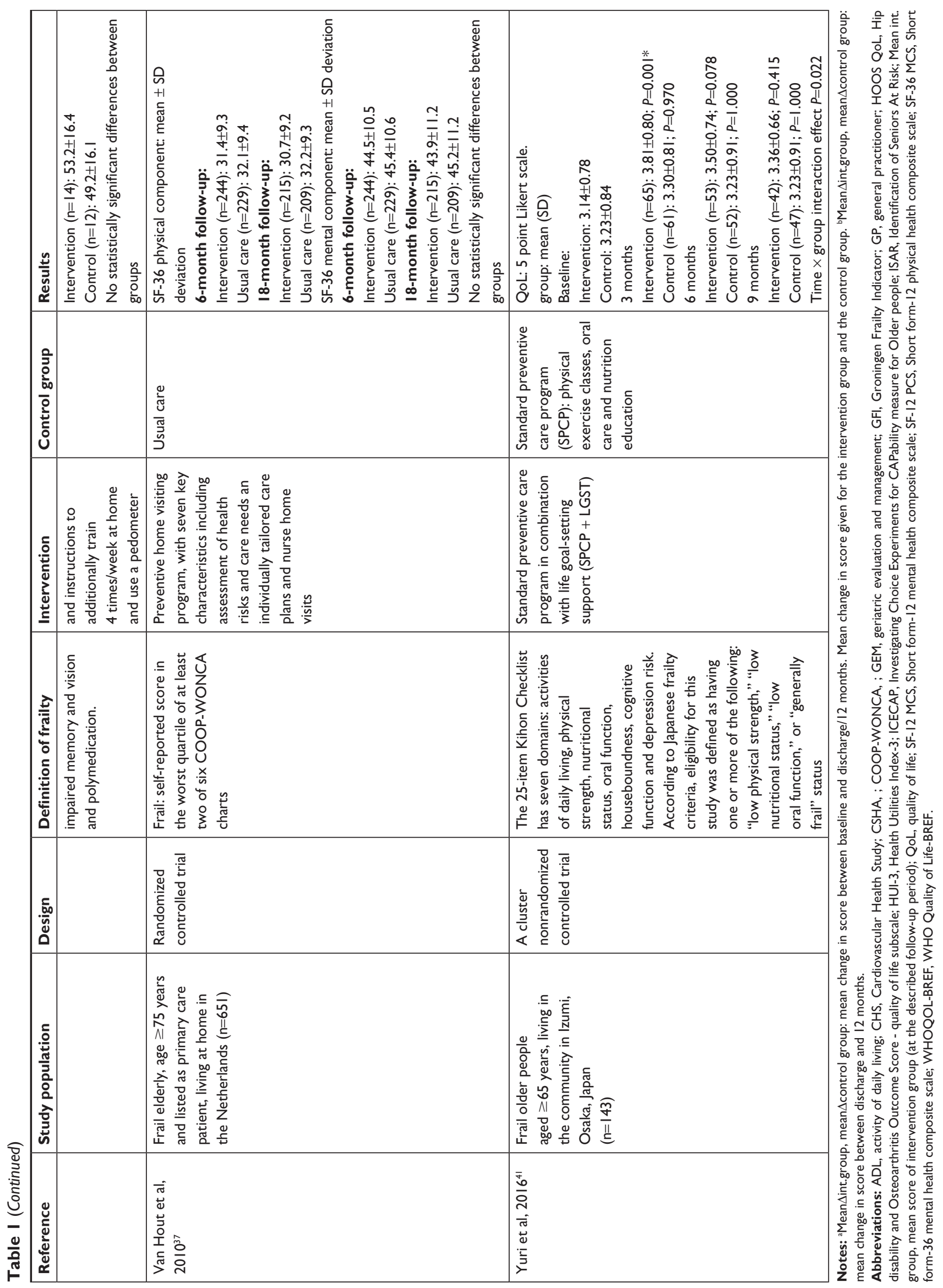


domain bodily pain was still present after one-year follow-up $(P=0.01)$. The outpatient geriatric care had a positive effect on: energy $(P=0.009)$, mental health $(P=0.001)$, and general health $(P=0.01)$. When mean changes of scores of the intervention and control group between discharge and 1-year follow-up were compared, only the improvement in the score for the mental health dimension remained significant $(P=0.004)$. Ekerstad et $\mathrm{a}^{40}$ reported a statistically significant difference between groups for four of the eight dimensions of the HUI-3 questionnaire (speech, ambulation, cognition and pain).

\section{Exercise programs}

Three of the six studies which examined the effect of an exercise program on quality of life reported no statistically significant difference between groups. ${ }^{33,35,36}$ Giné-Garriga et $\mathrm{al}^{32}$ reported a statistically significant difference between groups concerning the physical composite score (PCS) and the mental composite score (MCS) on the SF-12, after 12 and 36 weeks. After 12 weeks the average score of the PCS of the intervention group and the control group was 35.59 (SD 4.41) and 29.80 (SD 3.74) $(P<0.001)$ respectively. Langlois et $\mathrm{al}^{39}$ reported a statistically significant difference in favor of the intervention group in several domains of the Quality of Life Systemic Inventory questionnaire: global quality of life, leisure activities, perception of physical capacity, social/ family relationships, and perceived physical health. Yuri et $\mathrm{al}^{41}$ reported a statistically significant improvement in the quality of life measured with a five point Likert scale.

\section{Other interventions: testosterone gel, nurse home visits with alert buttons or acupuncture}

The study describing a testosterone gel intervention vs placebo gel reported a statistically significant difference on the somatic subscale 6 months after treatment $(P=0.04),{ }^{20}$ which was not sustained 12 months after treatment $(P=0.08) .{ }^{19,20}$ Favela et $\mathrm{al}^{31}$ examined the effect of 1 ) weekly nurse home visits in combination with alert buttons and 2) only nurse home visits, and reported that none of the interventions had a statistically significant effect on quality of life. Likewise, van Hout et $\mathrm{al}^{37}$ reported no statistically significant effect of nurse home visits on quality of life. Chan et $\mathrm{al}^{28}$ reported statistically significant improvement on several domains of the WHOQOL-BREF after an acupressure intervention.

\section{Discussion}

This systematized review provides an overview of the effects of different health care interventions for frail elderly on quality of life. The reported effects seem low, but the findings were inconsistent and the study designs were very heterogeneous in the design. Ten intervention studies reported no statistically significant difference between the intervention and control groups. Seven studies reported a statistically significant effect on subdomains of quality of life and two studies reported a statistically significant effect of the intervention on the overall quality of life score.

Two previous reviews have focused in particular on the effect of exercise interventions for frail elderly. ${ }^{42,43}$ One of these, the systematic review by Chou et al, ${ }^{42}$ concluded that exercise interventions had no effect on quality of life, but the results of the meta-analyses suggest that exercise increased gait speed, improved balance, and improved ADL performance of frail elderly. ${ }^{42}$ The other, a review by Theou et al, ${ }^{43}$ reported that exercise improved quality of life in four of the ten studies. The conclusions of these two reviews correspond with our results, namely inconclusive results concerning the effect of exercise interventions on frail elderly's quality of life.

Several possible explanations for the lack of evidence of the effectiveness of the health care interventions have been proposed by the authors of several studies included in this review. First, the intervention and control treatments might not be very different. As health professionals will already be aware of the risks involved in treating frail elderly patients, ${ }^{22}$ usual care often had many matching elements to the geriatric evaluation and management (GEM) intervention program and other multidisciplinary interventions. Therefore, the effectiveness of these interventions appeared to be limited. ${ }^{22,24-26,29,38}$ Second, inadequate implementation of an intervention will affect research results..$^{25,26,37,38}$ Several articles on the Dutch National Care Program for Elderly ${ }^{4-46}$ indicated that the efficacy of a multidisciplinary intervention aimed at frail older people depended on adequate implementation of the intervention. Inadequate implementation occurred when eg, only part of the protocol was followed, application of the full protocol was perceived as time consuming, the protocol was perceived as difficult or because health care professionals requiring more training to apply the protocol. ${ }^{25,37,38,47,48}$ During process evaluation of the multidisciplinary treatment program of Metzelthin et al, ${ }^{25}$ professionals did report that they did not always follow the whole protocol due to time constraints or its complexity. Adequate implementation of the protocol is not only dependent on the professional, but also on the frail elderly. If a protocol is time-consuming or difficult to perform for the elderly, inadequate implementation or loss to follow-up may be the result. Third, group size might be decisive for a 
study's results; two studies were pilot, randomized controlled trials with few participants. ${ }^{33,36}$ A similar trial with adequate sample sizes might result in different findings.

A major limitation of the present review was the heterogeneity of the included studies. The studies used different frailty measurements, different health care interventions and different outcome measurements for quality of life, which made it difficult to compare the results and to draw conclusions.

Furthermore, we focused only on the outcome quality of life. We did not describe adverse effects of the intervention. For that matter, most of the studies did not describe adverse effects. The testosterone gel intervention, however, is controversial. It is unclear if short-term testosterone treatment leads to long-term effects and there are concerns about its possible adverse effects, such as increased PSA and prostate cancer progression, as stated in the editorial comment on one of the articles. ${ }^{49}$ Furthermore, high intensity resistance exercise might lead to musculoskeletal injury. In the study of Latham et al, ${ }^{35}$ eighteen people (15\%) in the exercise group had musculoskeletal injuries compared with five (4\%) in the control group (RR 3.6, 95\% CI 1.5-8.0). The other studies did not describe any adverse effects. The strength of this review is that this study included all health care interventions, which resulted in an overview of all assessed health care interventions and their results.

\section{Conclusion}

In conclusion, this review found conflicting and inconsistent results concerning the effects of health care interventions for frail elderly on quality of life. Most of the included studies, however, reported no differences between intervention and control groups. As the population of older people will continue to grow, it will be important to pursue the search for effective health care interventions, not only regarding quality of life, but also other outcomes. Different aspects should be taken into consideration in order to make progression and improve quality of life among frail elderly in daily practice. It might be needed to adjust interventions enhancing the quality of the interventions and increasing the difference between the intervention and usual care. It is important to assure proper implementation of an intervention. It might be needed to adjust outcome measurements.

\section{Author contributions}

All authors contributed toward data analysis, drafting and critically revising the paper, gave final approval of the version to be published, and agree to be accountable for all aspects of the work.

\section{Disclosure}

The authors report no conflicts of interest in this work.

\section{References}

1. United Nations DoEaSA, Population Division. World Population Prospects: The 2015 Revision, Key Findings \& Advace Tables. New York: United Nations; 2015.

2. Lp F, Tangen CM, Walston J, et al. Frailty in older adults: evidence for a phenotype. J Gerontol. 2001;56(3):146-156.

3. Gobbens RJ, Luijkx KG, Wijnen-Sponselee MT, Schols JM. Towards an integral conceptual model of frailty. J Nutr Health Aging. 2010;14(3): 175-181.

4. Rodríguez-Mañas L, Féart C, Mann G, et al. Searching for an operational definition of frailty: a Delphi method based consensus statement. The frailty operative Definition-Consensus conference project. $J$ Gerontol A Biol Sci Med Sci. 2013;68(1):62-67.

5. de Vries NM, Staal JB, van Ravensberg CD, Hobbelen JS, Olde Rikkert MG, Nijhuis-van der Sanden MW. Outcome instruments to measure frailty: a systematic review. Ageing Res Rev. 2011;10(1):104-114.

6. Rockwood K, Stadnyk K, Macknight C, Mcdowell I, Hébert R, Hogan DB. A brief clinical instrument to classify frailty in elderly people. The Lancet. 1999;353(9148):205-206.

7. Fugate Woods N, Lacroix AZ, Gray SL, et al. Frailty: emergence and consequences in women aged 65 and older in the women's health Initiative Observational study. J Am Geriatr Soc. 2005;53(8):1321-1330.

8. Gobbens RJ, van Assen MA. Frailty and its prediction of disability and health care utilization: the added value of interviews and physical measures following a self-report questionnaire. Arch Gerontol Geriatr. 2012;55(2):369-379.

9. Rockwood K, Song X, Macknight C, et al. A global clinical measure of fitness and frailty in elderly people. CMAJ. 2005;173(5):489-495.

10. Bilotta $\mathrm{C}$, Bowling $\mathrm{A}$, Casè $\mathrm{A}$, et al. Dimensions and correlates of quality of life according to frailty status: a cross-sectional study on community-dwelling older adults referred to an outpatient geriatric service in Italy. Health Qual Life Outcome. 2010;8(1):56-65.

11. Lin CC, Ci L, Chang CK, et al. Reduces health-related quality of life in elders with frailty: a cross-sectional study of community-dwelling elders in Taiwan. PLoS One Journal. 2011;6(7):1-7.

12. Masel MC, Graham JE, Reistetter TA, Markides KS, Ottenbacher KJ. Frailty and health related quality of life in older Mexican Americans. Health Qual Life Outcome. 2009;770.

13. Kanwar A, Singh M, Lennon R, Ghanta K, Mcnallan SM, Roger VL. Frailty and health-related quality of life among residents of long-term care facilities. J Aging Health. 2013;25(5):792-802.

14. Kojima G, Iliffe S, Jivraj S, Walters K. Association between frailty and quality of life among community-dwelling older people: a systematic review and meta-analysis. J Epidemiol Community Health. 2016;70(7): 716-721.

15. Chang Y-W, Chen W-L, Lin F-G, et al. Frailty and its impact on healthrelated quality of life: a cross-sectional study on elder communitydwelling preventive health service users. PLoS One. 2012;7(5):e38079.

16. Gobbens RJ, van Assen MA, Luijkx KG, Schols JM. The predictive validity of the Tilburg frailty indicator: disability, health care utilization, and quality of life in a population at risk. The Gerontologist. 2012; 52(5):619-631.

17. Grant MJ, Booth A. A typology of reviews: an analysis of 14 review types and associated methodologies. Health Inform Lib J. 2009; 26(2):91-108.

18. Higgins JPT, Altman DG, Gotzsche PC, et al. The Cochrane collaboration's tool for assessing risk of bias in randomised trials. $B M J$. 2011;343(2):d5928. 
19. O'Connell MD, Roberts SA, Srinivas-Shankar U, et al. Do the effects of testosterone on muscle strength, physical function, body composition, and quality of life persist six months after treatment in intermediate-frail and frail elderly men? J Clin Endocrinol Metab. 2011;96(2):454-458.

20. Srinivas-Shankar U, Roberts SA, Connolly MJ, et al. Effects of testosterone on muscle strength, physical function, body composition, and quality of life in intermediate-frail and frail elderly men: a randomized, double-blind, placebo-controlled study. J Clin Endocrinol Metab. 2010;95(2):639-650.

21. Hempenius L, Slaets JPJ, van Asselt D, de Bock TH, Wiggers T, van Leeuwen BL. Long term outcomes of a geriatric liaison intervention in frail elderly cancer patients. PLoS One. 2016;11(2):e0143364.

22. Hempenius L, Slaets JPJ, van Asselt D, de Bock GH, Wiggers T, van Leeuwen BL. Outcomes of a geriatric liaison intervention to prevent the development of postoperative delirium in frail elderly cancer patients: report on a multicentre, randomized, controlled trial. PLoS One. 2013;8(6):e64834.

23. Looman WM, Fabbricotti IN, de Kuyper R, Huijsman R. The effects of a PRO-active integrated care intervention for frail community-dwelling older people: a quasi-experimental study with the GP-practice as single entry point. BMC Geriatr. 2016;16(1):43.

24. Looman WM, Fabbricotti IN, Huijsman R. The short-term effects of an integrated care model for the frail elderly on health, quality of life, health care use and satisfaction with care. Int J Integr Care. 2014;14(4):e034.

25. Metzelthin SF, Rossum E, Witte LP, et al. Frail elderly people living at home; effects of an interdisciplinary primary care programme. [Dutch] Nederlands tijdschrift voor geneeskunde. 2014;158(17):A7355.

26. Metzelthin SF, van Rossum E, Hendriks MR, et al. Reducing disability in community-dwelling frail older people: cost-effectiveness study alongside a cluster randomised controlled trial. Age Ageing. 2015;44(3): 390-396.

27. Cameron ID, Fairhall N, Langron C, et al. A multifactorial interdisciplinary intervention reduces frailty in older people: randomized trial BMC Med. 2013;11(1):65.

28. Chan CWC, Chau PH, Leung AYM, et al. Acupressure for frail older people in community dwellings - a randomised controlled trial. Age Ageing. 2017;46(6):957-964.

29. Cohen HJ, Feussner JR, Weinberger M, et al. A controlled trial of inpatient and outpatient geriatric evaluation and management. $\mathrm{N} \mathrm{Engl}$ J Med. 2002;346(12):905-912.

30. Fairhall N, Sherrington C, Kurrle SE, et al. Economic evaluation of a multifactorial, interdisciplinary intervention versus usual care to reduce frailty in frail older people. J Am Med Dir Assoc. 2015;16(1):41-48.

31. Favela J, La C, Franco-Marina F, et al. Nurse home visits with or without alert buttons versus usual care in the frail elderly: a randomized controlled trial. Clin Interventions Aging. 2013;8:85-95.

32. Giné-Garriga M, Guerra M, Unnithan VB. The effect of functional circuit training on self-reported fear of falling and health status in a group of physically frail older individuals: a randomized controlled trial. Aging Clin Exp Res. 2013;25(3):329-336.

33. Hoogeboom TJ, Dronkers JJ, van den Ende CHM, Oosting E, van Meeteren NLU. Preoperative therapeutic exercise in frail elderly scheduled for total hip replacement: a randomized pilot trial. Clin Rehabil. 2010;24(10):901-910.

34. Hoogendijk EO, van der Horst HE, van de Ven PM, et al. Effectiveness of a geriatric care model for frail older adults in primary care: results from a stepped wedge cluster randomized trial. Eur J Intern Med. 2016; $28: 43-51$.
35. Latham NK, Anderson CS, Lee A, et al. A randomized, controlled trial of quadriceps resistance exercise and vitamin $\mathrm{D}$ in frail older people: the frailty Interventions Trial in elderly subjects (fitness). J Am Geriatr Soc. 2003;51(3):291-299.

36. Oosting E, Jans MP, Dronkers JJ, et al. Preoperative home-based physical therapy versus usual care to improve functional health of frail older adults scheduled for elective total hip arthroplasty: a pilot randomized controlled trial. Arch Phys Med Rehabil. 2012;93(4):610-616.

37. van Hout HPJ, Jansen APD, van Marwijk HWJ, et al. Prevention of adverse health trajectories in a vulnerable elderly population through nurse home visits: a randomized controlled trial [ISRCTN05358495]. J Gerontol A: Biol Sci Med Sci. 2010;65A(7):734-742.

38. Kircher TTJ, Wormstall H, Muller PH, et al. A randomised trial of a geriatric evaluation and management consultation services in frail hospitalised patients. Age and Ageing. 2007;36(1):36-42.

39. Langlois F, Vu TTM, Chasse K, Dupuis G, Kergoat M-J, Bherer L. Benefits of physical exercise training on cognition and quality of life in frai older adults. J Gerontol B: Psychol Sci Soc Sci. 2013;68(3):400-404.

40. Ekerstad N, Dahlin-Ivanoff S, Landahl S, et al. Is the acute care of frail elderly patients in a comprehensive geriatric assessment unit superior to conventional acute medical care? Clin Interv Aging. 2017;12:1239-1249.

41. Yuri Y, Takabatake S, Nishikawa T, Oka M, Fujiwara T. The effects of a life goal-setting technique in a preventive care program for frail community-dwelling older people: a cluster nonrandomized controlled trial. BMC Geriatrics. 2016;16(1):1-11.

42. Chou C-H, Hwang C-L, Wu Y-T, Yt W. Effect of exercise on physical function, daily living activities, and quality of life in the frail older adults: a meta-analysis. Arch Phys Med Rehabil. 2012;93(2):237-244.

43. Theou O, Stathokostas L, Roland KP, et al. The effectiveness of exercise interventions for the management of frailty: a systematic review. J Aging Res. 2011;2011(3):1-19.

44. Fabbricotti IN, Janse B, Looman WM, de Kuijper R, van Wijngaarden JDH, Reiffers A. Integrated care for frail elderly compared to usual care: a study protocol of a quasi-experiment on the effects on the frail elderly, their caregivers, health professionals and health care costs. BMC Geriatrics. 2013;13(1):31.

45. Buurman BM, Parlevliet JL, Allore HG, et al. Comprehensive geriatric assessment and transitional care in acutely hospitalized patients: the transitional care bridge randomized clinical trial. JAMA Intern Med. 2016;176(3):302-309.

46. Asmus-Szepesi KJE, de Vreede PL, Nieboer AP, et al. Evaluation design of a reactivation care program to prevent functional loss in hospitalised elderly: a cohort study including a randomised controlled trial. $B M C$ Geriatrics. 2011;11(1):36.

47. de Vos A, Cramm JM, van Wijngaarden JDH, Bakker TJEM, Mackenbach JP, Nieboer AP. Understanding implementation of comprehensive geriatric care programs: a multiple perspective approach is preferred. Int J Health Plann Manag. 2017;32(4):608-636.

48. van Haastregt JCM, Diederiks JPM, van Rossum E, Dwl P, Crebolde H. Effects of preventive home visits to elderly people living in the community: systematic review. BMJ. 2000;320(7237):754-758.

49. Griebling TL. Re: do the effects of testosterone on muscle strength, physical function, body composition, and quality of life persist six months after treatment in intermediate-frail and frail elderly men? J Urol. 2012;187(2):622. 


\section{Supplementary material Embase and Medline (OvidSP) search strategies}

Embase (Embase and Medline)

('frail elderly'/de OR ((frail* AND (elder* OR old* OR aged OR geriatr* OR octogenar*)) OR (impair* NEXT/1 elder*)):ab,ti) AND ('quality of life'/exp OR ((qualit* NEAR/3 (life OR liv*)) OR QOL OR HRQL):ab,ti)
Medline (OvidSP)

("Frail Elderly"/ OR ((frail* AND (elder* OR old* OR aged OR geriatr* OR octogenar*)) OR (impair* ADJ elder*)). ab,ti.) AND ("Quality of Life"/ OR ((qualit* ADJ3 (life OR liv*)) OR QOL OR HRQL).ab,ti.)

\section{Publish your work in this journal}

Clinical Interventions in Aging is an international, peer-reviewed journal focusing on evidence-based reports on the value or lack thereof of treatments intended to prevent or delay the onset of maladaptive correlates of aging in human beings. This journal is indexed on PubMed Central, MedLine,
CAS, Scopus and the Elsevier Bibliographic databases. The manuscript management system is completely online and includes a very quick and fair peer-review system, which is all easy to use. Visit http://www.dovepress. com/testimonials.php to read real quotes from published authors. 